\title{
The Microstructure and Strength of a V-5Cr-5Ti Alloy Processed by High Pressure Torsion
}

Zhijian Fan ${ }^{\mathrm{a}, \mathrm{b}}$, Bertalan Jóni ${ }^{\mathrm{b}}$, Gábor Ribárik ${ }^{\mathrm{b}}$, Éva Ódor ${ }^{\mathrm{b}}$, Zs. Fogarassy $^{\mathrm{c}}$, Tamás Ungár ${ }^{\mathrm{b}, \mathrm{d}}$,

${ }^{a}$ Key Laboratory of Neutron Physics and Institute of Nuclear Physics and Chemistry, China Academy of Engineering Physics, Mianyang, 621999, China,

${ }^{\mathrm{b}}$ Department of Materials Physics, Eötvös University, Budapest, P.O.Box 32, H-1518, Hungary

${ }^{c}$ Research Institute for Technical Physics and Materials Science, Hungarian Academy of Science, Budapest, Hungary.

${ }^{\mathrm{d}}$ School of Materials, The University of Manchester, M13 9PL, Manchester, UK

\begin{abstract}
The microstructure and the strength of high pressure torsion processed $\mathrm{V}-5 \mathrm{Cr}-5 \mathrm{Ti}$ alloy are investigated by X-ray line profile analysis and microhardness testing. High pressure torsion has been applied at 4 and $8 \mathrm{GPa}$ with $0.25,0.5,1,2,4$ and 8 rotations. The $\mathrm{X}$-ray beam of the high angular resolution diffractometer, dedicated for line profile analysis, has a footprint of about $200 \mu \mathrm{m} \times 1.5 \mathrm{~mm}$ on the specimen. The diffraction patterns have been measured in the center, at half radius and close to the edge of the specimens. This technique has provided a large number of data of the microstructure and hardness as a function of strain up to about $\gamma \cong 300$. The dislocation density determined by X-ray line broadening is correlated and discussed in terms of the strength of the alloy using the Taylor equation.
\end{abstract}

Keywords: Microstructure of V-5Cr-5Ti alloy; Dislocation density; Dislocation arrangement parameter; Crystallite size; Grain size; Plastic deformation; Fusion reactor first wall material; $X$-ray line profile analysis

Communicating author: Tamás Ungár,ungar@ludens.elte.hu

\section{Introduction}

Vanadium alloys are one of the prospective structural materials in fusion reactors [1-13]. Vanadium has relatively low activation properties [1], high thermal stress factors [2] and relatively good radiation resistance with moderate swelling properties [5,8]. Embrittlement by transmuted helium [7] and enhanced creep induced by neutron irradiation [9-11] are, however, issues in $\mathrm{V}$ base alloys. Considerable efforts have been made to improve high temperature strength, ductility at low temperatures, thermal or irradiation induced creep resistance and degradation under irradiation environment $[9,12,14]$. For the purpose of forming fine-grained structure and dispersive $\mathrm{Ti}(\mathrm{CNO})$ particles in $\mathrm{V}-4 \mathrm{Cr}-4 \mathrm{Ti}$ alloy, Tyumentsev and coworkers 
[15] applied a technique of multiple multidirectional forge molding (MDFM) combined with thermo-mechanical treatment. It was found that at the final stage the grain size and the particle size of $\mathrm{Ti}(\mathrm{CNO})$ were in the range of 3-5 $\mu \mathrm{m}$ and 10-20 nm, respectively. Authors concluded that reducing of grain size and refinement of second phase particle size was due to high plastic strain imposed by the multi-directional forge molding procedure. It has been claimed that large dislocation activity and high defect concentration produced by severe plastic deformation activates fragmentation of second phase particles and nucleation of small recrystallized grains. Zheng et al. [16] suggested another procedure of mechanical alloying (MA) followed by hot isostatic pressing to produce V-4Cr-4Ti-1.5Y-0.3TiC alloys. They found that the grain size can be refined to smaller values around $\sim 0.35 \mu \mathrm{m}$ along with the formation of TiN and $\mathrm{Y}_{2} \mathrm{O}_{3}$ nano-particles. With increasing MA time the strength of the alloy is improved due to hardening contributions of solid solution, grain size and nano-particle dispersion.

In recent years high-pressure torsion (HPT) has received much attention to improve mechanical properties of materials. During the HPT procedure samples are imposed to a large torsional strain under high hydrostatic pressure in order to produce bulk nanostructured materials with exceptionally high strength. Some reports [17-21] have shown that the applied pressure is an important external factor for the formation of homogeneous microstructure in the entire specimen. With increasing the pressure, the microhardness value in the center of the specimen will be more close to that in the edge of the specimen after larger numbers of revolutions. In Reference [21] two kinds of $b c c$ metals, tantalum and niobium, were processed by HPT under different pressures and the microstructure was investigated by X-ray line profile analysis. It was shown that in the specimens deformed with higher pressure the dislocation density was smaller. This peculiar behavior was attributed to the larger vacancy concentration retained during deformation in the specimen when deformed at higher pressure. At unloading a larger number of dislocations were annihilated because of the enhanced diffusion due to the larger instantaneous vacancy concentration.

In the present work we investigate the processes of grain refinement and dislocation density evolution induced by HPT up to shear stress values of $\gamma=290$ at two different hydrostatic pressures of 4 and $8 \mathrm{GPa}$, respectively. We have also investigated the thermal stability of the deformed microstructure up to $1200{ }^{\circ} \mathrm{C}$. Microhardness was measured at different positions of the HPT deformed specimens and quasi stress-strain curves were obtained. X-ray diffraction patterns were measured at different positions of the HPT disks by a dedicated high-angular resolution X-ray diffractometer capable of micro-diffraction. The diffraction patterns were evaluated for dislocation density, crystallite size and dislocation arrangement parameters using the convolutional multiple whole profile (CMWP) line profile analysis procedure. The mechanical properties and the microstructure of the specimens were analyzed in terms of the Taylor equation.

\section{Experimental}

\subsection{Samples}


The HPT specimens of $10 \mathrm{~mm}$ diameter and $\sim 0.7 \mathrm{~mm}$ thickness were cut from the V-5Cr-5Ti alloy. The discs were machined from an annealed metal bar produced by the General Research Institute for Nonferrous Metals, China. The chemical composition of the specimens is given in Table 1. For the initial V-5Cr-5Ti specimens, the grain size was $\sim 200 \mu \mathrm{m}$ and the dislocation density was below about $10^{12} \mathrm{~m}^{-2}$. Two pressure values of 4 and $8 \mathrm{GPa}$ were selected for the HPT deformation at room temperature. These relatively large pressure values were selected in order to avoid slipping of the disks during HPT. Six different rotations, i.e. $0.25,0.5,1,2,4$ and 8 , were implemented at a speed of 0.2 rotation $/ \mathrm{min}$ at each of the two pressure values, respectively. Shear deformation, $\gamma$, as a function of the radius, $r$ in the HPT deformed disk has been evaluated as [22]:

$\gamma=\frac{r 2 \pi \chi}{l 360^{\circ}}$

where $\chi$ is the angle of rotation in degrees and $l$ is the thickness of the disk. Measurements carried out on HPT deformed specimens are denoted by the pressure, rotation and radial position. For example the notation $4 \mathrm{G}-2$ rot- $0.5 R$ means that the specimen was deformed by 2 rotations at $4 \mathrm{GPa}$ pressure and the measurement was done at half radius of the disk. In order to avoid surface damage by the HPT plunger the disks were polished and chemically etched before hardness and X-ray diffraction measurements.

\subsection{X-ray diffraction experiments}

X-ray diffraction measurements were carried out in a special high-resolution diffractometer dedicated to line profile analysis with a plane Ge (220) primary monochromator operated at the $\mathrm{Cu} \mathrm{K \alpha}$ fine-focus rotating copper anode (Rigaku, RA-MultiMax9) at $40 \mathrm{kV}$ and $100 \mathrm{~mA}$ [23]. The distance between the source and the monochromator was $240 \mathrm{~mm}$. A slit of $160 \mu \mathrm{m}$ was put before the monochromator to cut off the $\mathrm{K} \alpha_{2}$ component of the X-ray beam. With this arrangement the $\mathrm{Cu} \mathrm{K} \alpha_{1}$ beam on the specimen surface has a size of about $0.2 \times 1.5 \mathrm{~mm}$. The diffraction geometry is of parallel-beam type. With the stationary specimens the scattered radiation was registered by two flat imaging plates (IPs) with a linear spatial resolution of 50 $\mu \mathrm{m}$. The IPs were placed at a distance of $300 \mathrm{~mm}$ from the specimen covering the $2 \theta$ angular range between 38 and 148 degrees. The distance between the specimen and detector was selected such that the instrumental effect was always less than $10 \%$ of the physical broadening of the peaks. The diffraction patterns were obtained by integrating the intensity distributions along the corresponding Debye-Scherrer arcs on the IPs. Only the central parts of the arcs were used for the integration where the curvature does not affect line broadening. The X-ray beam was positioned on the specimen surface by using a low-depth-resolution microscope coupled to a television screen. The X-ray diffraction measurements were done at the center, at half radius and close to the edge of the samples, denoted as $0 \mathrm{R}, 0.5 \mathrm{R}$ and $1 \mathrm{R}$, respectively. Two more positions at a quarter and three-quarter radius, i.e. $0.25 \mathrm{R}$ and $0.75 \mathrm{R}$, were also measured on the specimens deformed by 4 and 8 rotations, respectively. 


\subsection{Microhardness measurements}

The strength of the HPT processed specimens has been investigated by measuring the Vickers hardness HV in a Zwick/Roell-ZH $\mu$-Indentec microhardness tester. HV measurements were done at the same positions of the specimens where the X-ray diffraction experiments were carried out. The HV values at the same radius, $\mathrm{HV}(r)$, were averaged and used to calculate the flow stress as a function or $r: \sigma(r)=\mathrm{HV}(r) / 3$. The flow stress $\sigma(r)$ is shown as a function of shear deformation in a quasi stress-strain curve in Fig. 1. The figure shows that the flow stress values of the specimens deformed at 4 and $8 \mathrm{GPa}$ are the same within experimental error with the same saturation value of about $1220( \pm 40) \mathrm{MPa}$.

\subsection{Electron microscopy and heating experiments}

The transmission electron microscope (TEM) studies were carried out in a Philips CM-20 electron microscope working at $200 \mathrm{KeV}$. TEM thin-foil specimens were prepared from the 4 and $8 \mathrm{GPa}$ specimens, each HPT deformed to about $\gamma=300$. After mechanical polishing the specimens were prepared by the conventional twin-jet electro polishing technique using a solution of 25 vol.\% nitric acid and $75 \mathrm{vol} \%$ methanol. Two typical TEM micrographs of the specimen deformed at $4 \mathrm{GPa}$ by 4 rotations and taken from the half-radius and from the disk edge region are shown along with the corresponding grain-size distribution bar diagrams in Fig. 2. The thermal stability of the microstructure was tested by heating experiments up to $1200{ }^{\circ} \mathrm{C}$. Three quarter pieces of the $4 \mathrm{GPa} 4$ rotation HPT deformed specimen were heated in a SETARAM DTA-92 type differential thermal analyzer in dry Ar atmosphere to 800, 1000 and $1200{ }^{\circ} \mathrm{C}$ and kept at each temperature for $30 \mathrm{~min}$. After heat treatment the specimens were cooled in the DTA equipment. Chemical etching was applied to remove the damaged surface layer before X-ray measurements for line broadening.

\subsection{Evaluation of the $X$-ray diffraction experiments}

Usually, HPT produces large dislocation densities and sub-micron grain size due to large torsional deformation. According to the kinematical theory of X-ray diffraction, small crystallite size and large lattice distortions are causing peak broadening in diffraction patterns [24]. The dislocation density and arrangement, the dislocation characters and the crystallite size have been evaluated by using the CMWP line profile analysis procedure [25-27]. The theoretical models of dislocation and crystallite size implemented in the CMWP are described briefly as follows. According to the Krivoglaz-Wilkens theory the mean square strain $\left\langle\varepsilon_{g, L}{ }^{2}\right\rangle$ in dislocated crystals is $[28,29]$ :

$\left\langle\varepsilon_{g, L}^{2}\right\rangle \cong \frac{\rho \bar{C} b^{2}}{4 \pi} f(\eta)$

where $g$ is the length of the diffraction vector, $L$ is the Fourier variable, $\rho$ and $b$ are the density and the Burgers vector of dislocations, $\bar{C}$ is the average dislocation contrast factor and $f(\eta)$ is the strain function. The $f(\eta)$ function describes the $L$ dependence of the mean-square-strain with $\eta=L / R_{\mathrm{e}}$, where $R_{\mathrm{e}}$ is the effective outer cut-off radius of dislocations. 
The dislocation contrast factors can be averaged over the permutations of $h k l$ if the sample is a polycrystalline or if all possible Burgers vectors are activated. For cubic crystals the average contrast factor can be given as [30]:

$\bar{C}=\bar{C}_{h 00}\left(1-q \mathrm{H}^{2}\right)$,

where $\bar{C}_{h 00}$ is the average contrast factor of the $h 00$ reflections and $\mathrm{H}^{2}=\left(h^{2} k^{2}+h^{2} l^{2}+k^{2} l^{2}\right) /\left(h^{2}+k^{2}+k^{2}\right)^{2}$. The $q$ parameter depends on the elastic constants of the crystal and the type of dislocations, and can be evaluated numerically [25,31]. The crystallite size, i.e. the coherent domain size, obtained from X-ray line broadening is evaluated by assuming log-normal size distribution given by the median, $m$ and the variance, $\kappa$ [32]. Experience has shown that the best correlation with grains size in TEM is obtained with the area average means crystallite size [33]:

$\langle\mathrm{x}\rangle_{\text {area }}=m \times \exp \left(2.5 \kappa^{2}\right)$.

Although the CMWP procedure can also provide the density of planar defects in the present case we did not use this option because the V-5Cr-5Ti alloy has a high stacking fault energy and no twinning was observed under quasi-static deformation conditions [34]. Typical CMWP evaluated diffraction patterns are shown in Fig. 3. The measured (open circles) and calculated (solid red lines) diffraction patterns of the V-5Cr-5Ti deformed at $4 \mathrm{GPa} \gamma=0.37$ and $8 \mathrm{GPa} \gamma=125$ as well as at $4 \mathrm{GPa} \gamma=37$ and held at $1200^{\circ} \mathrm{C}$ for $30 \mathrm{~min}$ are compared in Figs. $3 \mathrm{a}$ and $3 \mathrm{~b}$, respectively. In order to avoid overlapping the patterns are shown in logarithmic intensity scale and are shifted relative to each other.

\section{Results}

\subsection{Dislocation densities, dipole character parameters and crystallite size of the HPT deformed specimens}

Quantitative evaluation of line broadening was done by the CMWP procedure. The results of the crystallite size $d_{\mathrm{x}}$ and the dislocation density $\rho$ are shown in Fig. 4 for the samples after deformation at 4 and $8 \mathrm{GPa}$ pressures. The crystallite size decreases with shear strain up to a value of about $\gamma=15$. Beyond that the crystallite size remains nearly unchanged at a value of $d_{\mathrm{x}} \cong 31( \pm 2) \mathrm{nm}$, as shown in Fig. 4c. The TEM micrographs in Fig. 2 show that the grains are elongated in the shear direction, a typical grain morphology in HPT deformed specimens [35-37]. The grain size, $d_{\text {TEM }}$ is determined normal to the shear direction. It was obtained for strain values of $\gamma=74$ and $\gamma=145$ from the TEM micrographs in Figs. $2 \mathrm{a}$ and $2 \mathrm{~b}$ as $d_{\mathrm{TEM}}=44$ $( \pm 15) \mathrm{nm}$ and $d_{\mathrm{TEM}}=36( \pm 13) \mathrm{nm}$, respectively, as shown in Figs. 2c and 2d. respectively. The dislocation density increases and saturates only at a much larger strain than the crystallite size, i.e. it increases up to about $\gamma=75$ and saturates at a value of about $82 \times 10^{14} \mathrm{~m}^{-2}$. This is shown in Fig. $4 \mathrm{c}$, where $\rho$ and $d_{\mathrm{x}}$ are plotted together up to $\gamma=100$. The crystallite size stops decreasing while the dislocation density is still increasing with strain. A similar phenomenon was also observed earlier in HPT deformed $\mathrm{Nb}$ and $\mathrm{Ta}$ [21]. Comparing the values of the crystallite size and the dislocation density in the V-5Cr-5Ti specimens at 4 and $8 \mathrm{GPa}$ pressures, they are nearly the same within the experimental uncertainty. 
Eq. (3) shows that the contrast factors of dislocations in a polycrystal are given by the q parameter, the only variable in $\bar{C}$. Note that $\bar{C}_{h 00}$ is a scaling parameter not fitted in the CMWP procedure, however, they can be adjusted by the numerically calculated values given in reference [25]. The evolution of $\mathrm{q}$ and the dipole character parameter, $M$, versus strain are plotted in Fig. 5.

\subsection{Results of the heating experiment}

The dislocation density, $\rho$, the subgrain size, $d_{\mathrm{x}}$ and the dislocation dipole character parameter, $M$ in the initial state and after two high temperature annealings of the specimen HPT deformed at $4 \mathrm{GPa}$ to $\gamma=37$ are shown in Fig. 6 . Up to $800{ }^{\circ} \mathrm{C}$ the dislocation density and the subgrain size are unchanged within the experimental error. The $M$ value, however, decreases from $M=5.8( \pm 0.4)$ before heating to $M=1.6( \pm 0.2)$ at $800{ }^{\circ} \mathrm{C}$ (see Fig. 6).

\section{Discussion}

\subsection{Dislocation structure produced by large plastic deformation}

The qualitative effect of microstrain and crystallite size on line broadening can be well observed in the Williamson-Hall (WH) [38] and modified Williamson-Hall ( $m$ WH) [39] plots of the full widths at half maxima (FWHM) of diffraction peaks. If the elastic anisotropy in the materials is strong the FWHM values in the WH plot reveal strongly non-monotonous increase versus $K$, where $K=2 \sin \theta / \lambda$ [36]. The effect of elastic anisotropy can be rationalized in the $m$ WH plot by replacing $K$ by $K \sqrt{\bar{C}}$ [39]. In the $m$ WH plot the FWHM values usually follow a straight line where the slope is qualitatively proportional to a certain momentum of the microstrain, and the intercept at $K=0$ is inversely proportional to the crystallite size. The $\mathrm{WH}$ and $m \mathrm{WH}$ plots of the $\mathrm{V}-5 \mathrm{Cr}-5 \mathrm{Ti}$ specimens deformed at 4 or $8 \mathrm{GPa}$ to different rotations and measured on the disks at the center (black open triangles), at 0.5R (red open circles), and at $0.75 \mathrm{R}$ (blue open squares) positions are plotted in Figs. 7 a to $7 \mathrm{~d}$, respectively. The FWHM values in the $\mathrm{WH}$ plots almost follow a linear trend in accordance with the relatively weak elastic anisotropy of $\mathrm{V}$, i.e. $2 \mathrm{c}_{44} /\left(\mathrm{c}_{11}-\mathrm{c}_{12}\right) \cong 0.779$ [40]. In the $m \mathrm{WH}$ plots the same average contrast factors were used as provided by the CMWP evaluation. The slopes of the straight lines in these plots are increasing with deformation up to about $\gamma \cong 40$ while the intersections at $K=0$ are increasing in agreement with decreasing crystallite size within the same deformation range.

The two average grain size values, $d_{\text {TEM }}$, obtained from the size distribution histograms in Figs. $2 \mathrm{c}$ and $2 \mathrm{~d}$, are $44( \pm 15) \mathrm{nm}$ and $36( \pm 13) \mathrm{nm}$ at $\gamma=74$ and $\gamma=145$, respectively. Both TEM grain size values are larger than the saturation value of the subgrain size, $d_{\mathrm{x}} \cong 31( \pm 2) \mathrm{nm}$, given by X-ray diffraction. As shown by Figs. $4 \mathrm{a}$ and $4 \mathrm{c}$ the $\mathrm{X}$-ray subgrain size saturates at about $\gamma=15$. The TEM grain size, however, is still decreasing after $\gamma=15$ indicating that grain refinement proceeds while the subgrain size has already been saturated. The TEM grain size 
at $\gamma=145$ normal to the shear direction, i.e. $d_{\mathrm{TEM}}=36( \pm 13) \mathrm{nm}$, is almost identical to the subgrain size given by X-ray diffraction, i.e. $d_{\mathrm{x}} \cong 31( \pm 2) \mathrm{nm}$. The fact that the subgrain size saturates at a relatively early deformation stage at about $\gamma=15$ and that the TEM grain size approaches this value at $\gamma=145$ indicates that the lowest grain size attainable by plastic deformation at RT in this alloy is most probably around $31( \pm 2) \mathrm{nm}$.

In pure $f c c \mathrm{Cu}$ and $b c c \mathrm{Nb}$ and $\mathrm{Ta}$, the dislocation density was found to be smaller after HPT deformation at higher pressure [21]. The controversy was explained by the reduced vacancy mobility under large hydrostatic pressures [21]. It was argued that at higher hydrostatic pressure the excess vacancies and interstitials produced by annihilating dislocations are less mobile, therefore more abundant. As a consequence, when the specimen was unloaded, due to the instantaneously enhanced mobility of more trapped point defects, dislocations annihilated more effectively in the higher pressure specimen [21]. In the present HPT experiments we could not observe any remarkable differences in the dislocation densities of specimens deformed either at 4 or $8 \mathrm{GPa}$. Fig. $4 \mathrm{~b}$ shows that the dislocation densities saturate around the same level of about $82 \times 10^{14} \mathrm{~m}^{-2}$, within experimental error, for both pressure values. This result indicates that in the $\mathrm{V}-5 \mathrm{Cr}-5 \mathrm{Ti}$ alloy even an enhanced point defect concentration cannot easily annihilate dislocations created by plastic deformation. The heating experiments, discussed below, show a fairly stable microstructure up to about $800{ }^{\circ} \mathrm{C}$ in correlation with the relatively stable dislocation density discussed here.

\subsection{Dislocation character and arrangement}

Strain anisotropy of peak broadening is accounted for by the average dislocation contrast factor, $\bar{C}$. It is given by Eq. (3), where $q$ is the physical parameter fitted in CMWP. As discussed above, $q$ depends on the edge or screw character of dislocations and the elastic anisotropy of the crystal. According to the numerical calculations [25,31,41], in the $a / 2<111>\{110\}$ slip system the theoretical values of $q$ are -1.4 and 1.7 for the edge and screw dislocations, respectively. As shown in Fig. 6, the experimental values of $q$ vary in the range of -1.4 and 0 , which indicates that the majority of dislocations are of edge character. The results are in good correlation with the earlier work on $b c c \mathrm{Nb}$ and Ta, where also edge dislocation character was found to be dominant after HPT deformation [21]. Solute atoms can also shift the dislocation character towards edge type as it was found in mechanically alloyed $\mathrm{Al}-\mathrm{Mg}$ [42]. With increasing the nominal Mg content the dislocation character was shifting towards edge type. This finding is in correlation with stronger pinning of edge dislocations by solute atoms. In the present $\mathrm{V}-5 \mathrm{Cr}-5 \mathrm{Ti}$ alloy substantial $\mathrm{Cr}$ and $\mathrm{Ti}$ additions are in solid solution preferentially pinning edge dislocations. The dislocation arrangement parameter, $M=R_{\mathrm{e}} \sqrt{\rho}$, describing the dipole character of dislocations, is shown $v s . \gamma$ in Fig. 6b. Smaller $M$ values correspond to stronger dipole character [29,43]. The value of $M$ parameter decreases rapidly to a level of about $M=6( \pm 1)$ with increasing strain. The result indicates that the dipole character of dislocations becomes somewhat stronger compared to the initial state of the sample, however, even at large strains it does not become really significant. This behavior is in good correlation with the TEM micrographs in Fig. 2 which show that in this specimen 
there is no dislocation cell formation. In pure $\mathrm{Cu}$ which is a typical dislocation cell forming metal the $M$ value can reach around $M \cong 1$ when persistent slip bands are forming [44].

\subsection{Thermal stability of the dislocation structure}

The dislocation density, $\rho$, the subgrain size, $d_{\mathrm{x}}$ and the dipole character parameter, $M$ in the initial state and after two high temperature annealings of the specimen HPT deformed at 4 GPa to $\gamma=37$ are shown in Fig. 6 . Up to $800{ }^{\circ} \mathrm{C}$ the dislocation density and the subgrain size are unchanged within the experimental error. The $M$ value, however, decreases from $M=5.8$ $( \pm 0.4)$ before heating to $M=1.6( \pm 0.2)$ at $800{ }^{\circ} \mathrm{C}$ indicating that the dipole character becomes stronger at heating to this temperature. With stronger dipole character the screening of strain fields is more efficient and the corresponding stored energy becomes smaller [45,46]. Wilkens showed that when the $M$ parameter is smaller the tails of diffraction peaks become longer $[29,43,44]$. Fig. 8 a shows the intensity distributions of the 211 reflections of the specimen before heating (blue curve) and heated to $800{ }^{\circ} \mathrm{C}$ (red curve). In order to see the difference in the shape of the two profiles the intensities are normalized to the maximum and the breadths to the FWHM, respectively. The tail of the annealed specimen-peak is considerably longer than that of the initial specimen-peak in good correlation with the theoretically calculated peak profiles shown in figure (2) in reference [43] (see Fig. 8a).

The $m \mathrm{WH}$ plot of as deformed, the 800 and $1000{ }^{\circ} \mathrm{C}$ annealed specimens are shown in Fig. $8 \mathrm{~b}$. The slope of the FWHM values of the as-deformed specimen is much larger than that of the $800{ }^{\circ} \mathrm{C}$ one, although the CMWP calculated dislocation densities of the two specimens are the same within experimental error. The slope in the $m \mathrm{WH}$ plot is proportional to the microstrain which obviously depends on both the dislocation density and the character of strain fields of dislocations [43]. In the present case, in the as-deformed state the dipole character is considerably weaker than after annealing at $800{ }^{\circ} \mathrm{C}$. During annealing at $800{ }^{\circ} \mathrm{C}$ the dislocation arrangement is changing while the dislocation density remains unchanged. The dipoles character increases, i.e. the strain field of dislocations becomes more of short range type. The process can be considered intermediate towards full recovery which, however, occurs only above $800{ }^{\circ} \mathrm{C}$.

\subsection{Correlation between the strength and the microstructure}

As shown in Fig. 4, large plastic deformation causes the simultaneous increase of the dislocation density and the reduction of crystallite size. Work hardening proceeds up to about $\gamma \cong 50$, the dislocation density increases up to about $\gamma \cong 75$ and the decrease of crystallite size stops at about $\gamma \cong 15$ as shown in Figs. 1 and 4, respectively. This indicating that the leading mechanism in work hardening is dislocation accumulation. We correlate the flow stress with the dislocation density in Taylor's equation [47]:

$\sigma=\sigma_{0}+\alpha G \mathrm{~b} M_{\mathrm{T}} \sqrt{\rho}$, 
where $\sigma_{0}$ is the yield stress of the coarse grain and dislocation-free material, $\alpha$ is a constant between zero and unity, $G$ is the shear modulus and $M_{\mathrm{T}}$ is the Taylor factor for polycrystalline specimen. The best match between the measured and calculated flow stress values were obtained with $\sigma_{0}=523( \pm 20) \mathrm{MPa}$ and $\alpha=0.20( \pm 0.1)$ where $G, b$ and $M_{\mathrm{T}}$ were taken as $G=47$ $\mathrm{GPa}$ [40], $b=0.2624 \mathrm{~nm}$ and $M_{\mathrm{T}}=3$. The calculated versus measured flow stress values, $\sigma_{\text {calc }}$ vs. $\sigma_{\text {meas }}$, are shown in Fig. 9.

\section{Conclusions}

The evolution of the dislocation density and grain size along with work hardening has been determined in the $\mathrm{V}-5 \mathrm{Cr}-5 \mathrm{Ti}$ alloy a perspective material for future nuclear power applications. High resolution X-ray diffraction and line profile analysis has been used to determine the dislocation density and subgrain size during large deformations applied by the method of HPT and two different pressures. TEM micrographs have been used to determine the grain size and size distribution.

Heating experiments were applied to determine the thermal stability of the microstructure of the alloy. We found that work hardening proceeds up to about $\gamma \cong 50$ where it saturates at a level of about $\sigma \cong 1220( \pm 40) \mathrm{MPa}$. The dislocation density was obtained to increase up to about $\gamma \cong 75$ where it saturated at a level of about $\rho \cong 82( \pm 8) \times 10^{14} \mathrm{~m}^{-2}$. The subgrain size decreases to about $d_{\mathrm{x}} \cong 31( \pm 2) \mathrm{nm}$ at about $\gamma \cong 15$ and saturates at higher values. The heating experiments have revealed that the dislocation density remains fairly stable up to about 800 ${ }^{\circ} \mathrm{C}$. Diffraction peak shape and dislocation arrangement or dipole character analysis has shown that up to about $800{ }^{\circ} \mathrm{C}$ the dislocation line density stays constant while the dipole character becomes stronger revealing the onset of recovery process where the dislocation dipoles become narrower, however, are annealed either at a higher temperature or during longer holding times than $30 \mathrm{~min}$. Our study has shown that large plastic deformation of the $\mathrm{V}-5 \mathrm{Cr}-5 \mathrm{Ti}$ alloy produces a strongly strained microstructure and substantial work hardening revealing a relatively good temperature stability up to about $800{ }^{\circ} \mathrm{C}$.

\section{Acknowledgements}

Z.F. is grateful to the National Natural Science Foundation of China (Grant No. 11775195). G.R. gratefully acknowledges the support of the János Bolyai Research Fellowship of the Hungarian Academy of Sciences. T.U. is grateful for the EPSRC Leadership Fellowship [EP/I005420/1] for partial support of this work.

\section{References}

[1] S.J. Zinkle, H. Matsui, D.L. Smith, A.F. Rowcli, E. van Osch, K. Abe, V.A. Kazakov, J. Nucl. Mater. 258-263 (1998) 205-214.

[2] T. Muroga, M. Gasparotto, S.J. Zinkle, Overview of materials research for fusion 
reactors, Fusion Eng. Des. 61-62 (2002) 13-25.

[3] T. Muroga, T. Nagasaka, K. Abe, V.M. Chernov, H. Matsui, D.L. Smith, Z.-Y. Xu, S.J. Zinkle, Vanadium alloys - overview and recent results, J. Nucl. Mater. 307-311 (2002) 547-554.

[4] E.E. Bloom, S.J. Zinkle, F.W. Wiffen, Materials to deliver the promise of fusion power-progress and challenges, J. Nucl. Mater. 329-333 (2004) 12-19.

[5] R.J. Kurtz, K. Abe, V.M. Chernov, D.T. Hoelzer, H. Matsui, T. Muroga, G.R. Odette, Recent progress on development of vanadium alloys for fusion, J. Nucl. Mater. 329-333 (2004) 47-55

[6] S.J. Zinkle, Advanced materials for fusion technology, Fusion Eng. Des. 74 (2005) 3140.

[7] T. Muroga, Vanadium Alloys for Fusion Blanket Applications, Mater. Transact. 46 (2005) 405-411.

[8] S. J. Zinkle, Structural materials for fission \& fusion energy, Mater. Today, 12 (2009) 12-19.

[9] J.M. Chen, V.M. Chernov, R.J. Kurtz, T. Muroga, Overview of the vanadium alloy researches for fusion reactors, J. Nucl. Mater. 417 (2011) 289-294.

[10] T. Muroga, Vanadium for nuclear systems, Compr. Nucl. Mater. 4 (2012) 391-406.

[11] S. J. Zinkle, A. Möslang, Evaluation of irradiation facility options for fusion materials research and development, Fusion Eng. Des. 88 (2013) 472- 482.

[12] T. Muroga, J.M. Chen, V.M. Chernov, R.J. Kurtz, M. Le Flem, Present status of vanadium alloys for fusion applications, J. Nucl. Mater. 455 (2014) 263-268.

[13] J. Knaster, A. Moeslang, T. Muroga, Materials research for fusion, Nature Phys. 12 (2016) 424-434.

[14] H.Y. Fu, J.M. Chen, P.F. Zheng, T. Nagasaka, T. Muroga, Z.D. Li, S. Cui, Z.Y. Xu, Fabrication using electron beam melting of a $\mathrm{V}-4 \mathrm{Cr}-4 \mathrm{Ti}$ alloy and its thermo-mechanical strengthening study, J. Nucl. Mater. 442 (2013) S336-S340.

[15] A.N. Tyumentsev, I.A. Ditenberg, K.V. Grinyaev, VM. Chernov, M.M. Potapenko, Multi-directional forge molding as a promising method of enhancement of mechanical properties of V-4Ti-4Cr alloys, J. Nucl. Mater. 413 (2011) 103-106.

[16] P.F. Zheng, T. Nagasaka, T. Muroga, J.M. Chen, Microstructures and mechanical properties of mechanically alloyed $\mathrm{V}-4 \mathrm{Cr}-4 \mathrm{Ti}$ alloy dispersion strengthened by nano-particles, Fusion Eng. Des. 89 (2014) 1648-1652.

[17] A.P. Zhilyaev, G.V. Nurislamova, B.-K. Kim, M.D. Baró, J.A. Szpunar, T.G. Langdon, Experimental parameters influencing grain refinement and microstructural evolution during high-pressure torsion, Acta Mater. 51 (2003) 753-765.

[18] T. Ungár L. Balogh, Y.T. Zhu, Z. Horita, C. Xu, T.G. Langdon, Using X-ray microdiffraction to determine grain sizes at selected positions in disks processed by high-pressure torsion, Mater. Sci. Eng. A 444 (2007) 153-156.

[19] A.P. Zhilyaev, T.G. Langdon, Using high-pressure torsion for metal processing: Fundamentals and applications, Prog. Mater. Sci. 53 (2008) 893-979.

[20] E. Schafler, Effects of releasing the hydrostatic pressure on the nanostructure after severe plastic deformation of Cu, Scripta Mater. 62 (2010) 423-426.

[21] B. Jóni, E. Schafler, M. Zehetbauer, G. Tichy, T. Ungár, Correlation between the 
microstructure studied by X-ray line profile analysis and the strength of high-pressure-torsion processed Nb and Ta, Acta Mater. 61 (2013) 632-642.

[22] W. Skrotzki, A. Eschke, B. Jóni, T. Ungar, L.S. Toth, Yu. Ivanisenko, L. Kurmanaeva, New experimental insight into the mechanisms of nanoplasticity, Acta Mater. 61 (2013) 7271-7284.

[23] T. Ungar, S. Ott, P.G. Sanders, A. Borbely, J.R. Weertman, Dislocations, grain size and planar faults in nanostructured copper determined by high resolution $\mathrm{x}$-ray diffraction and a new procedure of peak profile analysis, Acta Mater. 46 (1998) 3693-3699.

[24] B.E. Warren, X-ray studies of deformed metals, Prog. Metal. Phys. 8 (1959) 147-202.

[25] T. Ungár, I. Dragomir, Á. Révész, A. Borbély, The contrast factors of dislocations in cubic crystals: the dislocation model of strain anisotropy in practice, J. Appl. Cryst. 32 (1999) 992-1002.

[26] T. Ungár, G. Ribárik, L. Balogh, Defect-related physical-profile-based X-ray and neutron line profile analysis, Met. Mater. Transact. A41 (2010) 1202-1209.

[27] G. Ribárik, B. Jóni, T. Ungár, Global optimum of microstructure parameters in the CMWP line-profile analysis method by combining Marquardt-Levenberg and Monte-Carlo procedures, J. Mater. Sci. Technol. 35 (2019) 1508-1514.

[28] M.A. Krivoglaz, in X-ray and neutron diffraction in non-ideal crystals, Springer, Berlin, New York, 1996.

[29] M. Wilkens, Theoretical aspects of kinematical X-ray diffraction profiles from crystals containing dislocation distributions, in: NBS Fundamental Aspects of Dislocation Theory, Spec. Publ. 317, II. Eds. J.A. Simmons, R. de Wit, R. Bullough, Washington, DC, USA, 1970, pp. 1195-1221.

[30] T. Ungár, G. Tichy, The effect of dislocation contrast on X-ray line profiles in untextured polycrystals, Phys. Stat. Sol. A, 147 (1999) 425-434.

[31] A. Borbély, J. Dragomir-Cernatescu, G. Ribárik, T. Ungár, Computer program ANIZC for the calculation of diffraction contrast factors of dislocations in elastically anisotropic cubic, hexagonal and trigonal crystals, J. Appl. Cryst. 36 (2003) 160-162.

[32] W.C. Hinds, Aerosol technology: properties, behavior and measurement of airborne particles. Wiley, New York, 1982.

[33] T. Ungár, G. Tichy, J. Gubicza, R. J. Hellmig, Correlation between subgrains and coherently scattering domains, J. Powder Diffr. 20 (2005) 366-375.

[34] L.-J. Gui, Y.-L. Liu, First-principles studying the properties of oxygen in vanadium: Thermodynamics and tensile/shear behavior, Comp. Cond. Matter, 7 (2016) 7-13.

[35] T. C. Lowe, R. Z. Valiev, The Use of Severe Plastic Deformation Techniques in Grain Refinement, J. Min. Met. Mater. Soc. JOM, 56 (2004) 64-68.

[36] L. S. Toth, C. Gu, Ultrafine-grain metals by severe plastic deformation, Mater. Charact. 92 (1914) 1-14.

[37] V. V. Stolyarov, Y. T. Zhu, I. V. Alexandrov, T. C. Lowe, R. Z. Valiev, Grain refinement and properties of pure Ti processed by warm ECAP and cold rolling, Mater. Sci. Eng., A343 (2003) 43-50

[38] G.K. Williamson, W.H. Hall, X-ray line broadening from filed aluminium and wolfram, Acta Metall. 1 (1953) 22-31.

[39] T. Ungár, A. Borbély, The effect of dislocation contrast on x-ray line broadening: A new 
approach to line profile analysis, Appl. Phys. Lett. 69 (1996) 3173-3175.

[40] D. I. Bolef, R. E. Smith, J. G. Miller, Elastic Properties of Vanadium. I. Temperature Dependence of the Elastic Constants and the Thermal Expansions, Phy. Rev, B15 (1971) 4100-4108.

[41] I.C. Dragomir, T. Ungár, The dislocations contrast factors of cubic crystals in the Zener constant range between zero and unity, J. Powder Diffr. 17 (2002) 104-111.

[42] J. Gubicza, M. Kassem, G. Ribárik, T. Ungár, The microstructure of mechanically alloyed $\mathrm{Al}-\mathrm{Mg}$ determined by X-ray diffraction peak profile analysis, Mater. Sci. Eng. A 372 (2004) 115-122.

[43] M. Wilkens, The Determination of Density and Distribution of Dislocations in Deformed Single Crystals from Broadened X-Ray Diffraction Profiles, Phys. Stat. Sol. a2 (1970) 359-370.

[44] M. Wilkens, K. Herz, H. Mughrabi, An X-ray diffraction study of cyclically and unidirectionally deformed copper single crystal, Z. Metallkde. 71 (1980) 376-384.

[45] M. Wilkens, Das Mittlere Spannungsquadrat $\left\langle\sigma^{2}\right\rangle$ Begrenzt Regellos Verteilter Verstzungen in Einem Zilinderfoermigen Koerper, Acta Metall., 17 (1969) 1155-1159.

[46] M. Wilkens, The mean square stress $\left\langle\sigma^{2}\right\rangle$ for a completely random and a restrictedly random distribution of dislocations in a cylindrical body, in: NBS Fundamental Aspects of Dislocation Theory, Spec. Publ. 317, II. Eds. J.A. Simmons, R. de Wit, R. Bullough, Washington, DC, USA, 1970, pp. 1191-1193.

[47] G.I. Taylor, The mechanism of plastic deformation of crystals. Part I. - Theoretical, Proc. Roy. Soc. A 145 (1934) 362-404.

Table 1. The chemical composition of the V-5Cr-5Ti specimens in wt\%.

\begin{tabular}{llllllllll}
\hline Element & $\mathrm{Cr}$ & $\mathrm{Ti}$ & $\mathrm{Fe}$ & $\mathrm{Cu}$ & $\mathrm{Si}$ & $\mathrm{O}$ & $\mathrm{N}$ & $\mathrm{C}$ & $\mathrm{V}$ \\
\hline Composition & 5.06 & 4.94 & $\leq 0.02$ & $\leq 0.005$ & $\leq 0.03$ & $\leq 0.03$ & $\leq 0.01$ & $\leq 0.01$ & left \\
\hline
\end{tabular}

\section{Captions}

Fig. 1. (a) Quasi stress-strain curves of the V-5Cr-5Ti specimens deformed by applying 4 (open red circles) and $8 \mathrm{GPa}$ (open blue triangles) pressures. (b) Zoomed $\gamma$ range up to $\gamma=100$ showing the first part of work hardening. The dashed lines are to guide the eye. Error bars are shown as thick vertical lines.

Fig. 2. TEM micrographs of the specimens deformed to $\gamma=74$ (a) and $\gamma=145$ (b). Grain size distributions from the two micrographs are in (c) and (d). The numbers in brackets in (c) and (d) are the standard deviations of the average grain size values. 
Fig. 3. The measured (open circles) and the fitted (solid red lines) diffraction patterns.

Comparison of diffraction patterns of specimens deformed at $4 \mathrm{GPa} \gamma=0.37$ and $8 \mathrm{GPa} \gamma=125$ as well as the sample deformed at $4 \mathrm{GPa} \gamma=37$ and held at $1200{ }^{\circ} \mathrm{C}$ for $30 \mathrm{~min}$, (a) is the first and (b) the second half of the diffraction patterns, respectively. The patterns are shown in logarithmic intensity scale and are shifted relative to each other to avoid overlapping.

Fig. 4. (a) Area average mean crystallite size $d_{\mathrm{x}}$ and (b) dislocation density $\rho$ of the V-5Cr-5Ti specimens after deformation at 4 and 8 GPa pressures $v s . \gamma$. (c) The $d_{\mathrm{x}}$ and $\rho$ values $v s . \gamma$ up to $\gamma=100$ for the specimen deformed at $4 \mathrm{GPa}$. Error bars are shown as thick vertical lines.

Fig. 5. (a) The $q$ parameter, (b) the $M$ parameter of the V-5Cr-5Ti specimens after deformation at 4 and $8 \mathrm{GPa}$ pressures $v s$. the shear strain $\gamma$. Error bars are shown as thick vertical lines. The dotted horizontal lines in (a) indicate the q values for pure edge or screw character dislocations, respectively.

Fig. 6. Results of the heating experiment. (a) Dislocation density, $\rho$, subgrain size, $d_{\mathrm{x}}$ and the dipole character parameter, $M$ of $4 \mathrm{GPa} 4$ rotation HPT deformed specimen after $30 \mathrm{~min}$ holding at $\mathrm{T}$.

Fig. 7. WH (a) and mWH (b) plots of the FWHM after deformation at 4 GPa pressure. WH (c) and $m \mathrm{WH}(\mathrm{d})$ plots of the FWHM after deformation at $8 \mathrm{GPa}$ pressure.

Fig. 8. (a) Intensity distributions of the 211 reflections of the $4 \mathrm{GPa} \gamma=37$ deformed specimen (red curve) and after heated to $800{ }^{\circ} \mathrm{C}$ (blue curve) with logarithmic intensity scale normalized to the maximum and the FWHM. (b) mWH plots of the FWHM for the same three states of the alloy as shown in Fig. 6.

Fig. 9. Calculated, $\sigma_{\text {calc }} v s$. the measured, $\sigma_{\text {meas }}$, flow-stress values. The thick black vertical line indicates the error. 
(a)
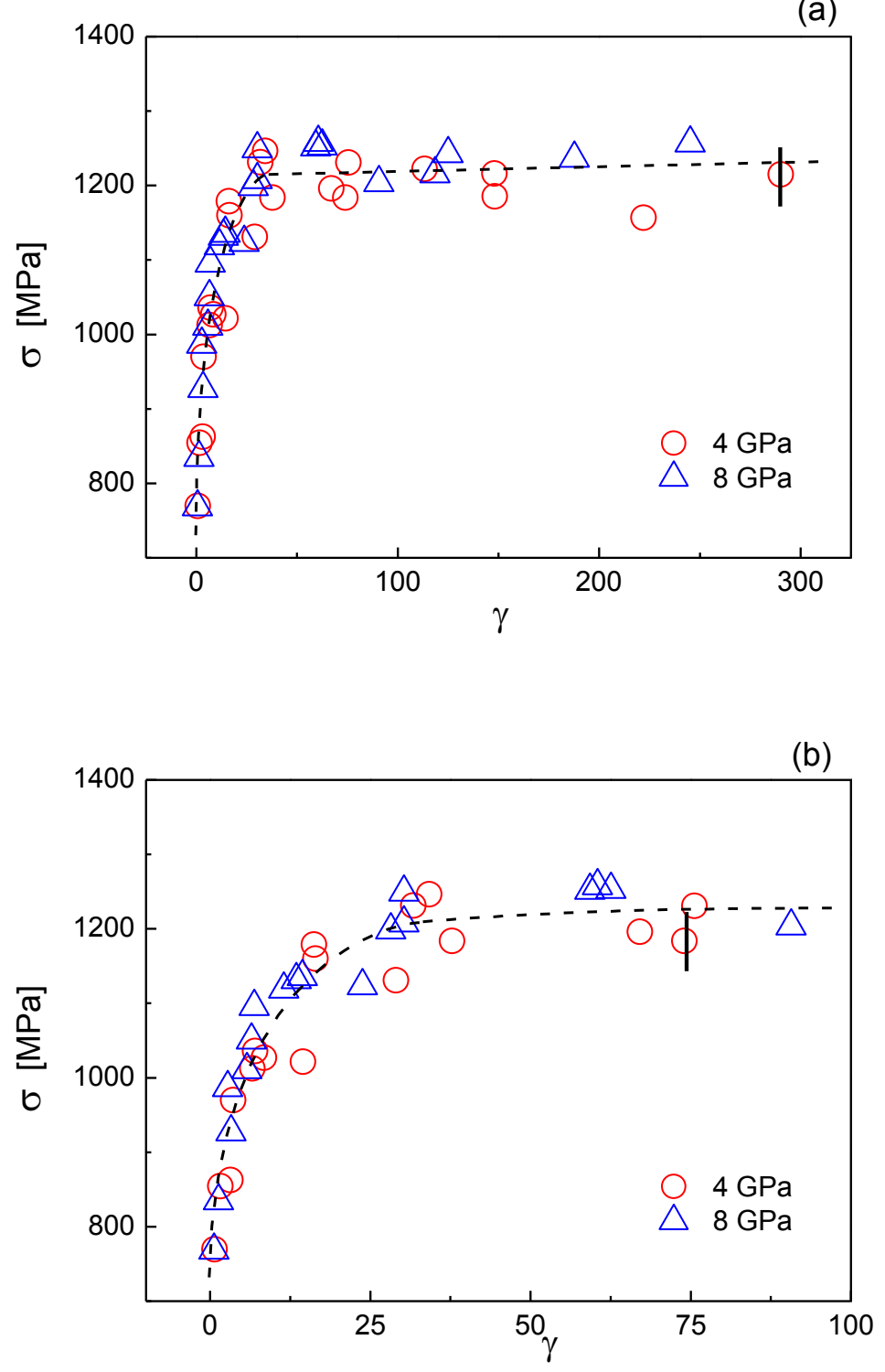

Fig. 1. (a) Quasi stress-strain curves of the V-5Cr-5Ti specimens deformed by applying 4 (open red circles) and $8 \mathrm{GPa}$ (open blue triangles) pressures. (b) Zoomed $\gamma$ range up to $\gamma=100$ showing the first part of work hardening. The dashed lines are to guide the eye. Error bars are shown as thick vertical lines. 


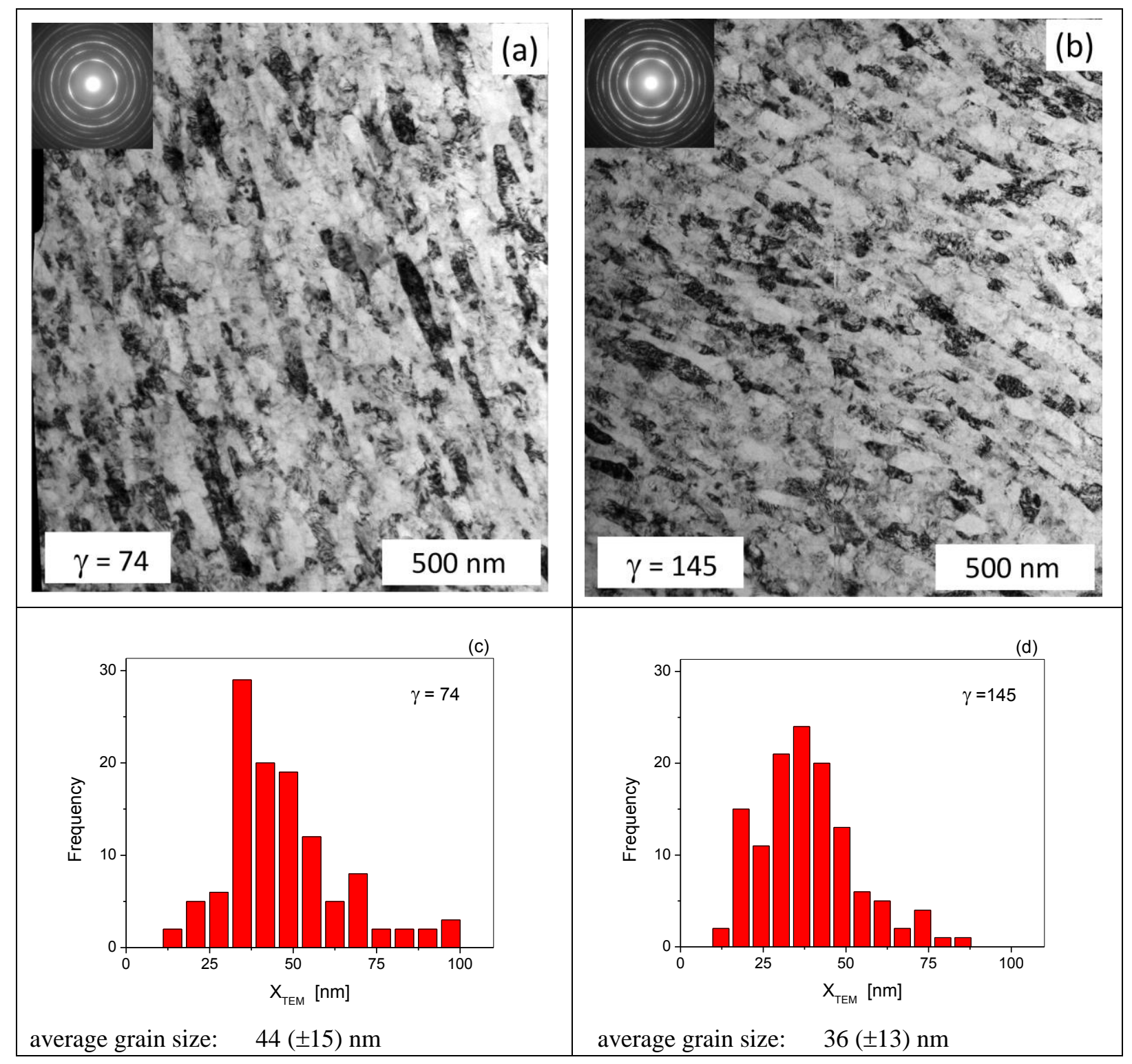

Fig. 2. TEM micrographs of the specimens deformed to $\gamma=74$ (a) and $\gamma=145$ (b). Grain size distributions from the two micrographs are in (c) and (d). The numbers in brackets in (c) and (d) are the standard deviations of the average grain size values. 
(a)

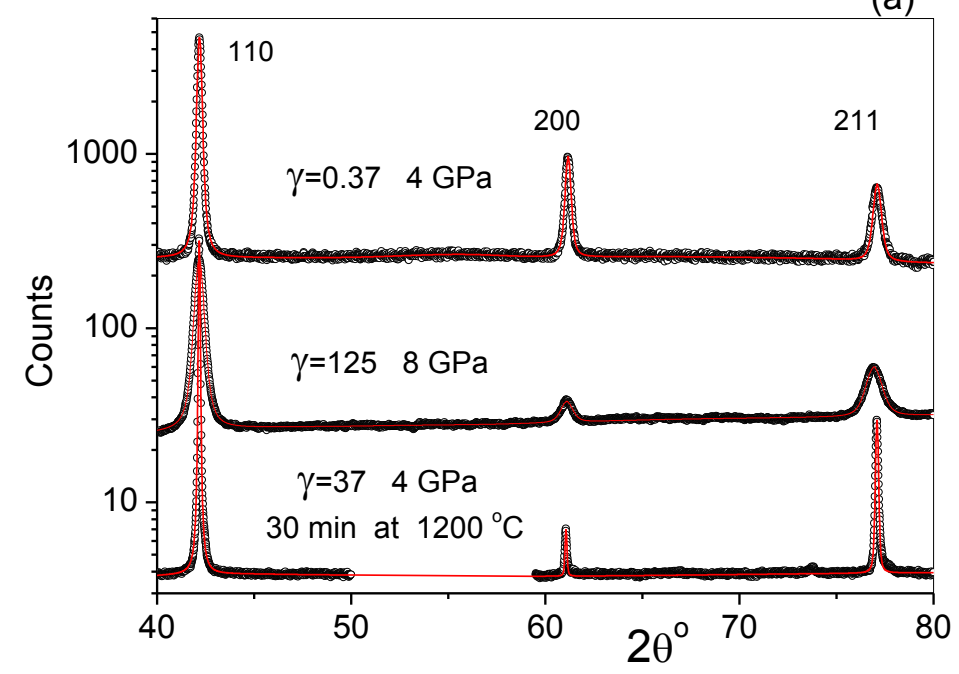

(b)

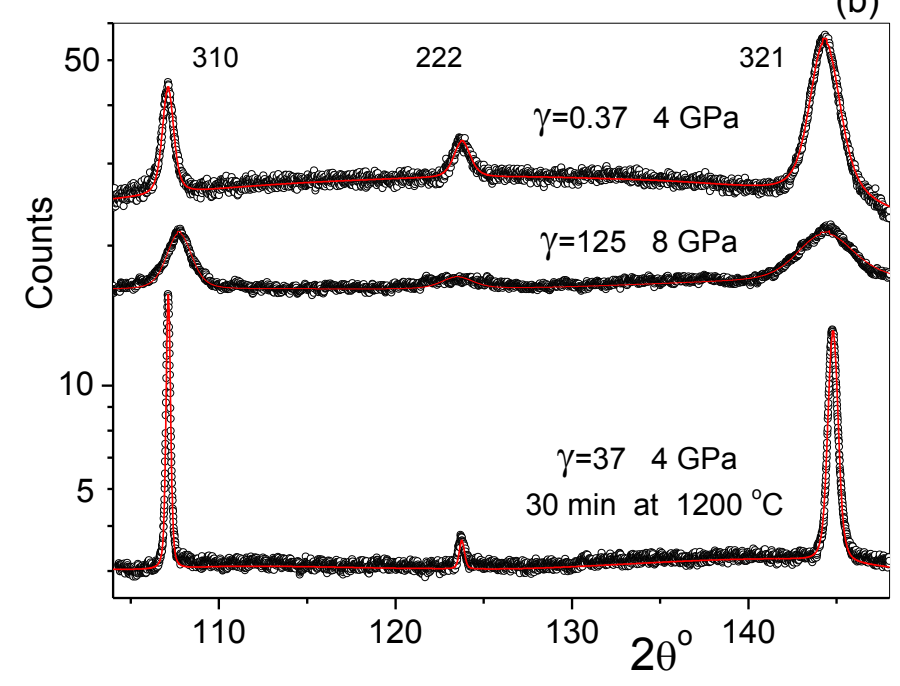

Fig. 3. The measured (open circles) and the fitted (solid red lines) diffraction patterns. Comparison of diffraction patterns of specimens deformed at $4 \mathrm{GPa} \gamma=0.37$ and $8 \mathrm{GPa} \gamma=125$ as well as the sample deformed at $4 \mathrm{GPa} \gamma=37$ and held at $1200{ }^{\circ} \mathrm{C}$ for $30 \mathrm{~min}$, (a) is the first and (b) the second half of the diffraction patterns, respectively. The patterns are shown in logarithmic intensity scale and are shifted relative to each other to avoid overlapping. 

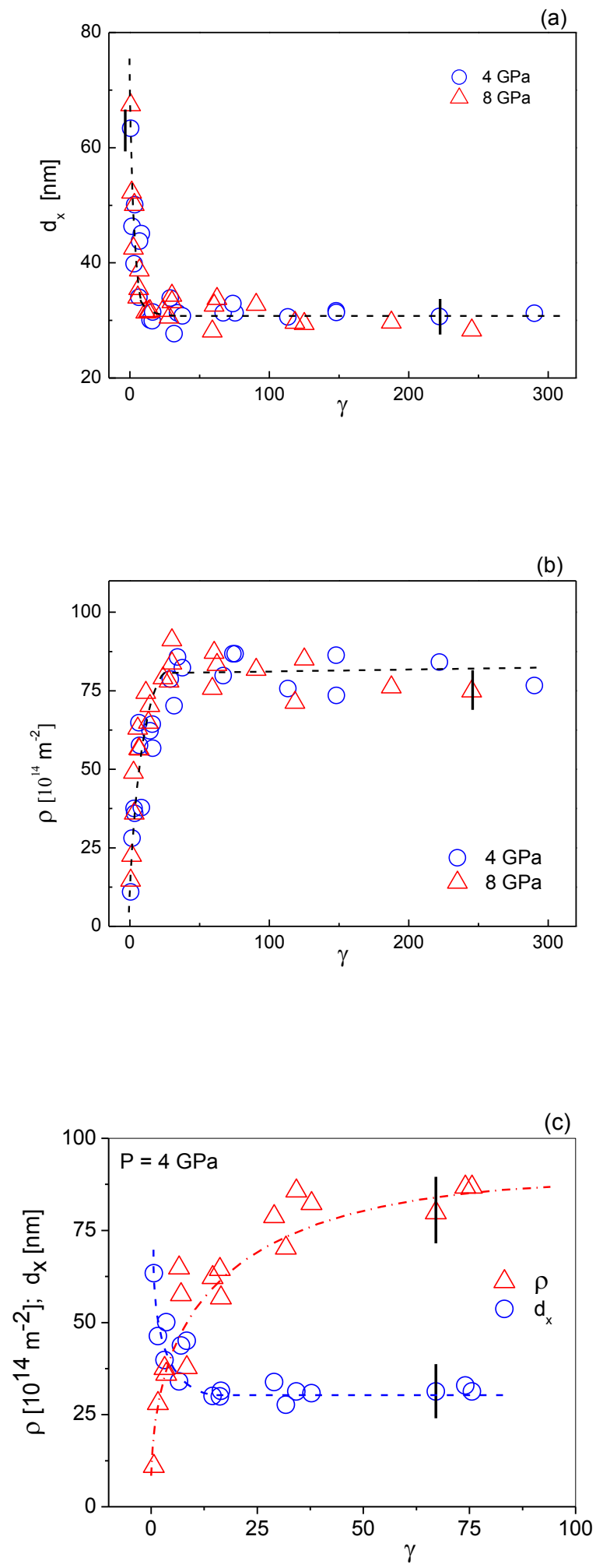

Fig. 4. (a) Area average mean crystallite size $d_{\mathrm{x}}$ and (b) dislocation density $\rho$ of the V-5Cr-5Ti specimens after deformation at 4 and 8 GPa pressures $v s$. $\gamma$. (c) The $d_{\mathrm{x}}$ and $\rho$ values $v s . \gamma$ up to $\gamma=100$ for the specimen deformed at $4 \mathrm{GPa}$. Error bars are shown as thick vertical lines. 
(a)

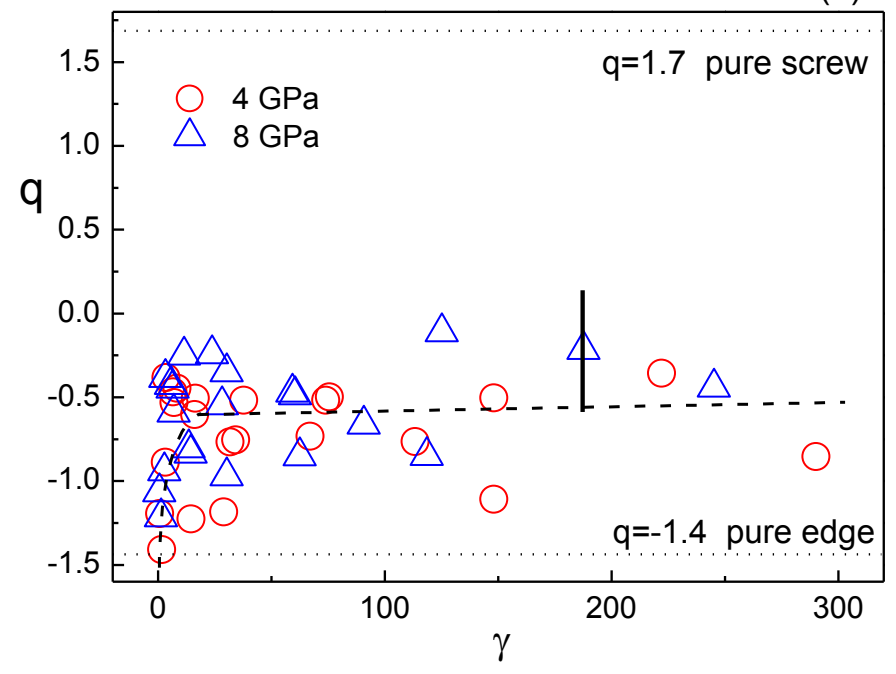

(b)

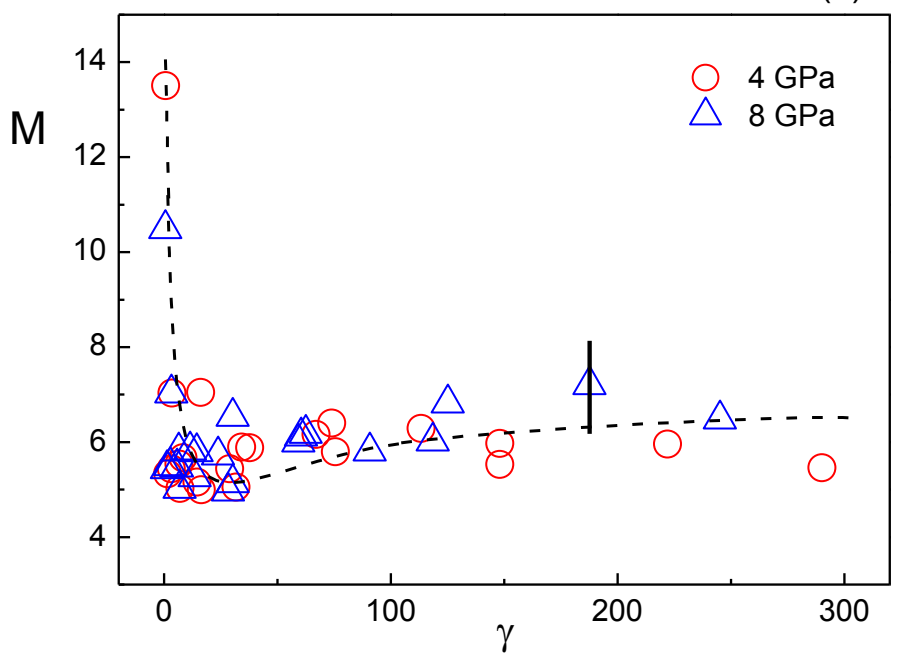

Fig. 5. (a) The $q$ parameter, (b) the $M$ parameter of the $\mathrm{V}-5 \mathrm{Cr}-5 \mathrm{Ti}$ specimens after deformation at 4 and $8 \mathrm{GPa}$ pressures $v s$. the shear strain $\gamma$. Error bars are shown as thick vertical lines. The dotted horizontal lines in (a) indicate the q values for pure edge or screw character dislocations, respectively. 


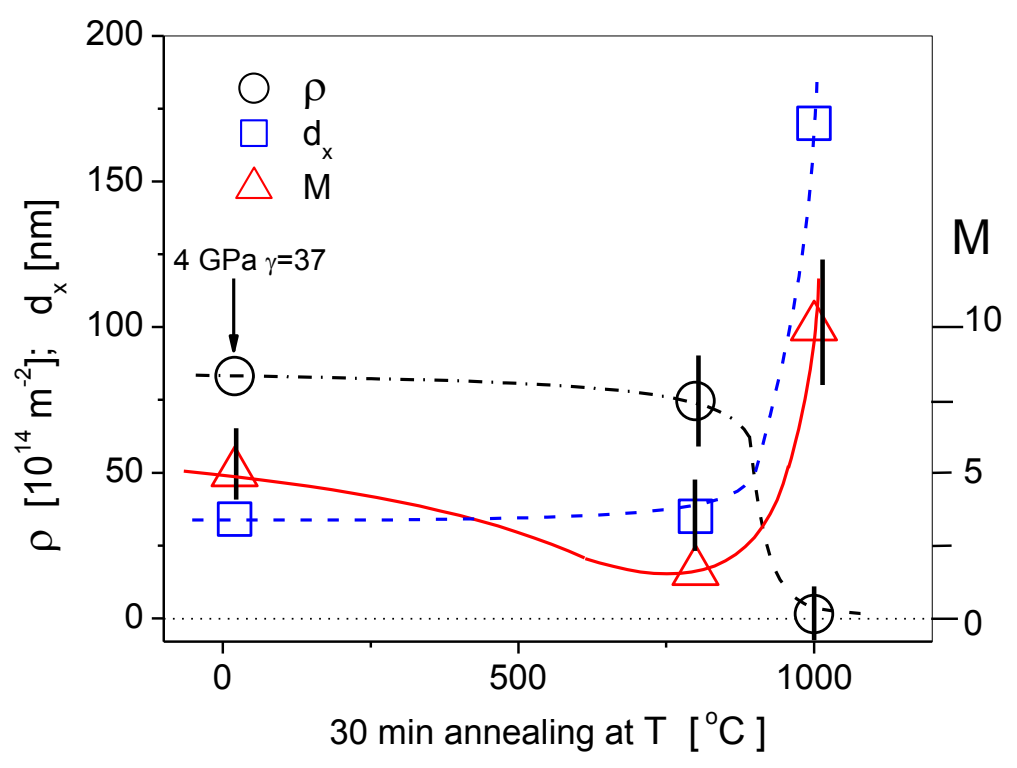

Figure 6. Results of the heating experiment. (a) Dislocation density, $\rho$, subgrain size, $d_{\mathrm{x}}$ and the dipole character parameter, $M$ of $4 \mathrm{GPa} 4$ rotation HPT deformed specimen after 30 min holding at $\mathrm{T}$. 

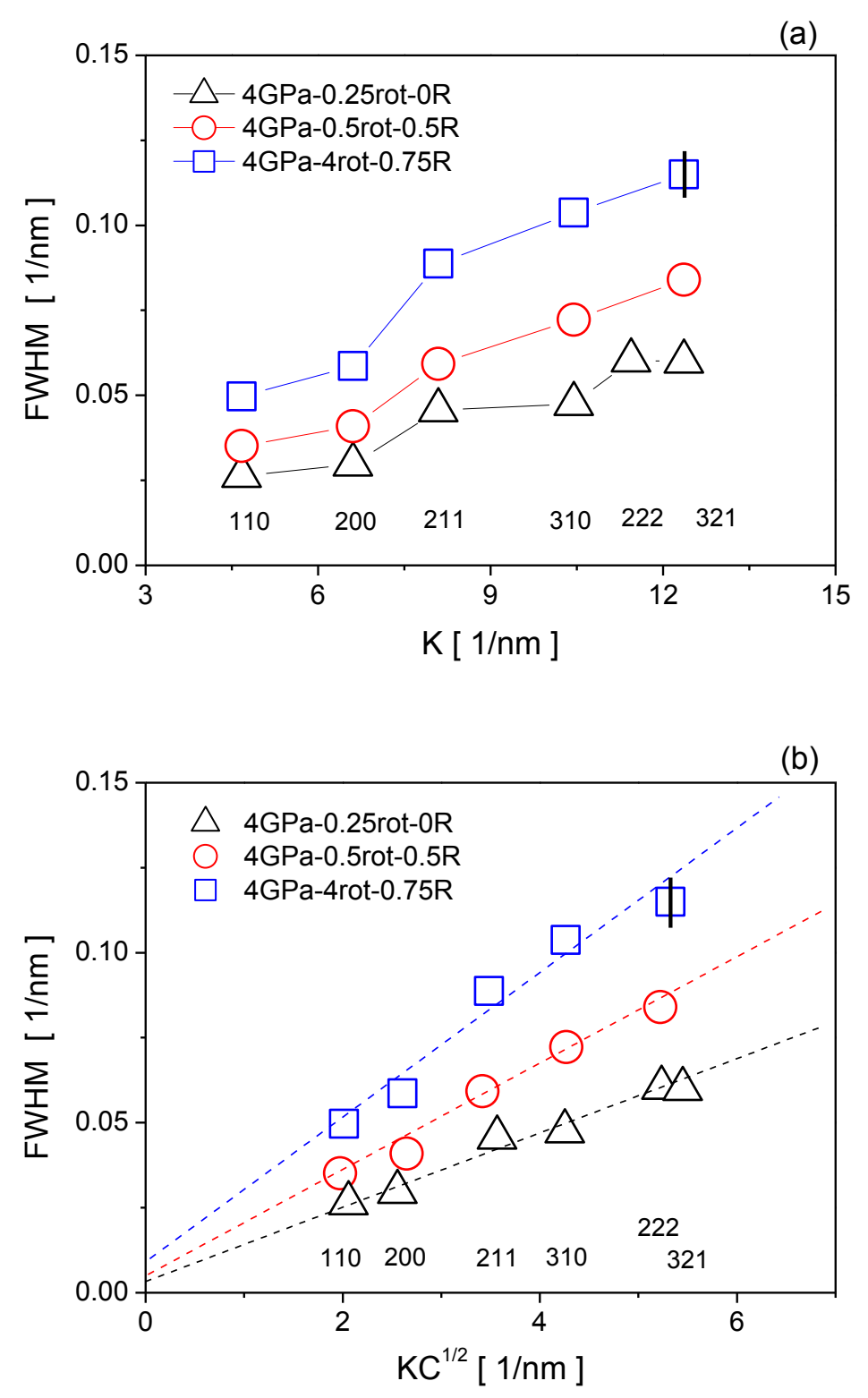

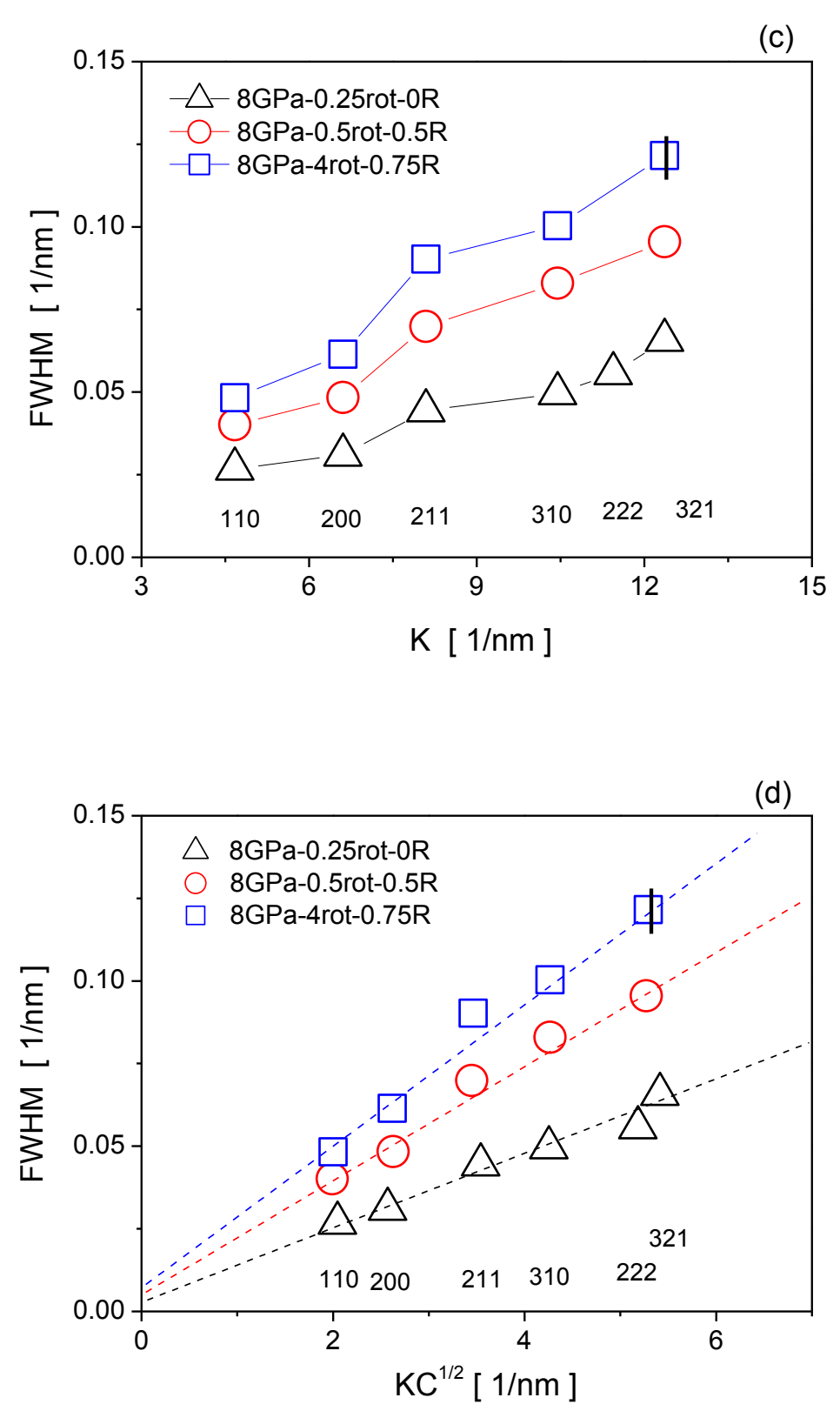

Fig. 7. WH (a) and mWH (b) plots of the FWHM after deformation at 4 GPa pressure. WH (c) and $m \mathrm{WH}(\mathrm{d})$ plots of the FWHM after deformation at $8 \mathrm{GPa}$ pressure. 
(a)
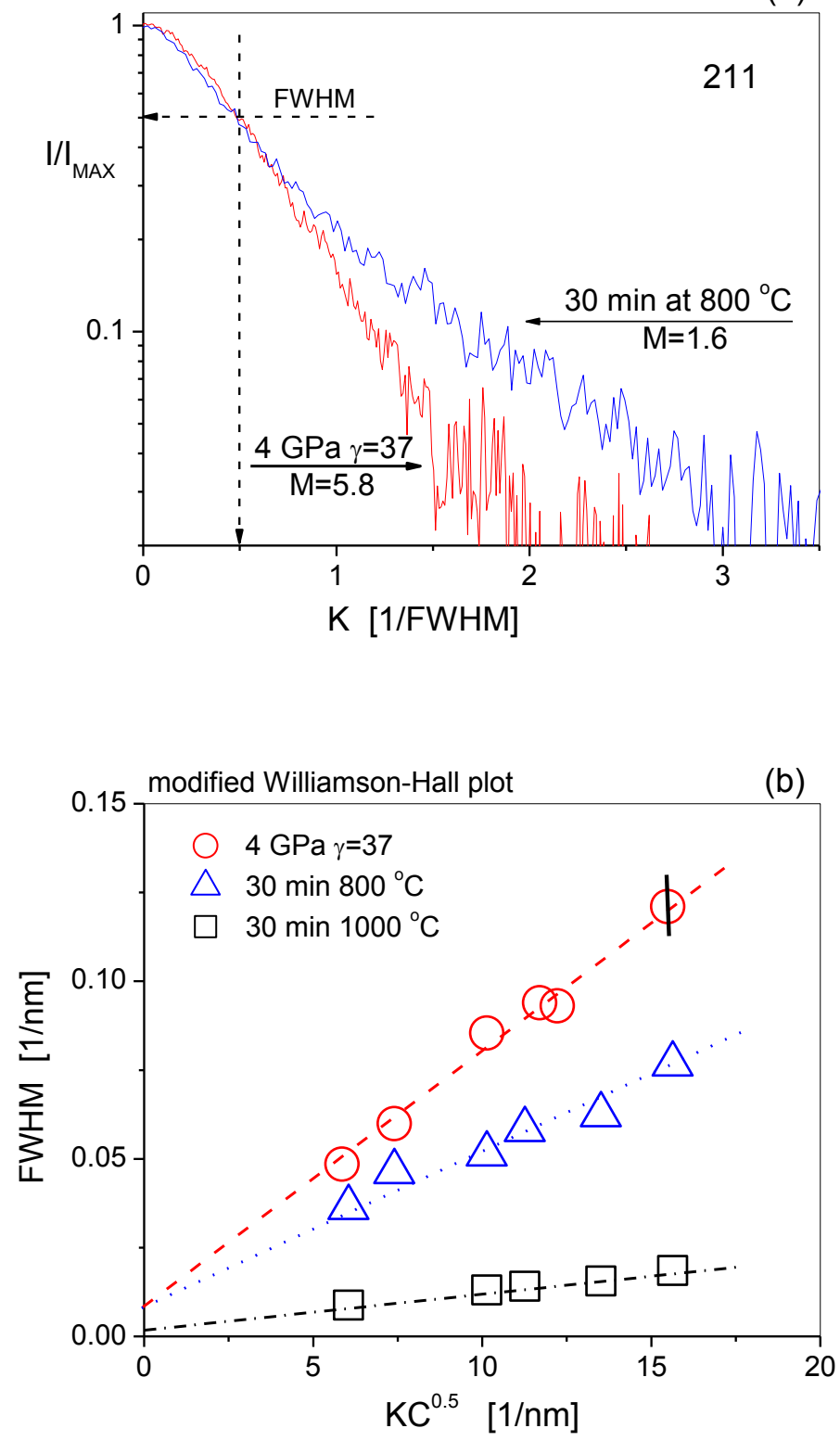

Fig. 8. (a) Intensity distributions of the 211 reflections of the $4 \mathrm{GPa} \gamma=37$ deformed specimen (red curve) and after heated to $800{ }^{\circ} \mathrm{C}$ (blue curve) with logarithmic intensity scale normalized to the maximum and the FWHM. (b) mWH plots of the FWHM for the same three states of the alloy as shown in Fig. 6. 


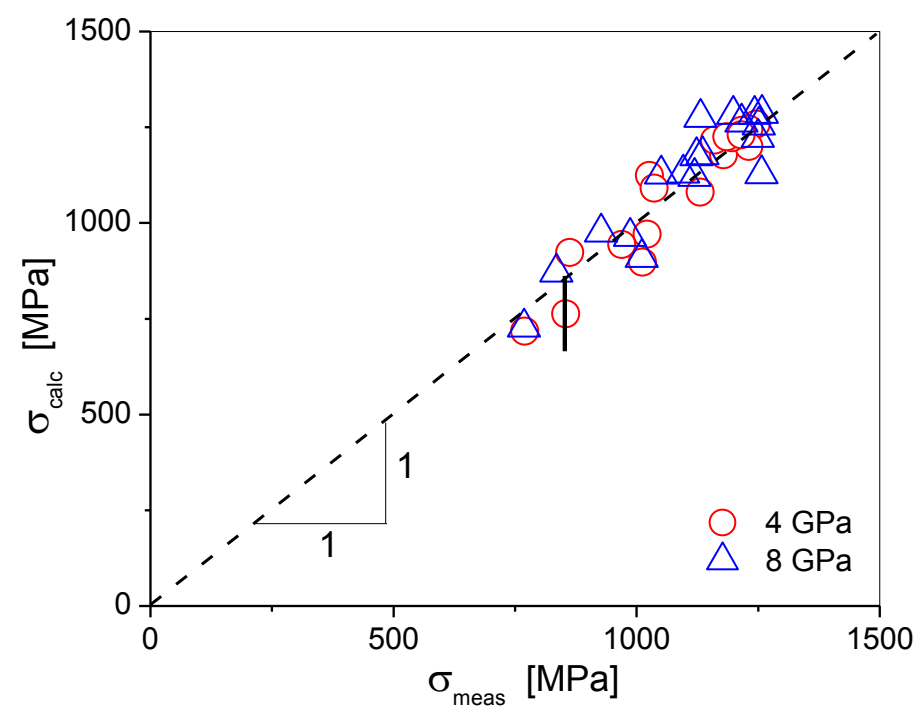

Fig. 9. Calculated, $\sigma_{\text {calc }} v s$. the measured, $\sigma_{\text {meas }}$, flow-stress values. The thick black vertical line indicates the error. 